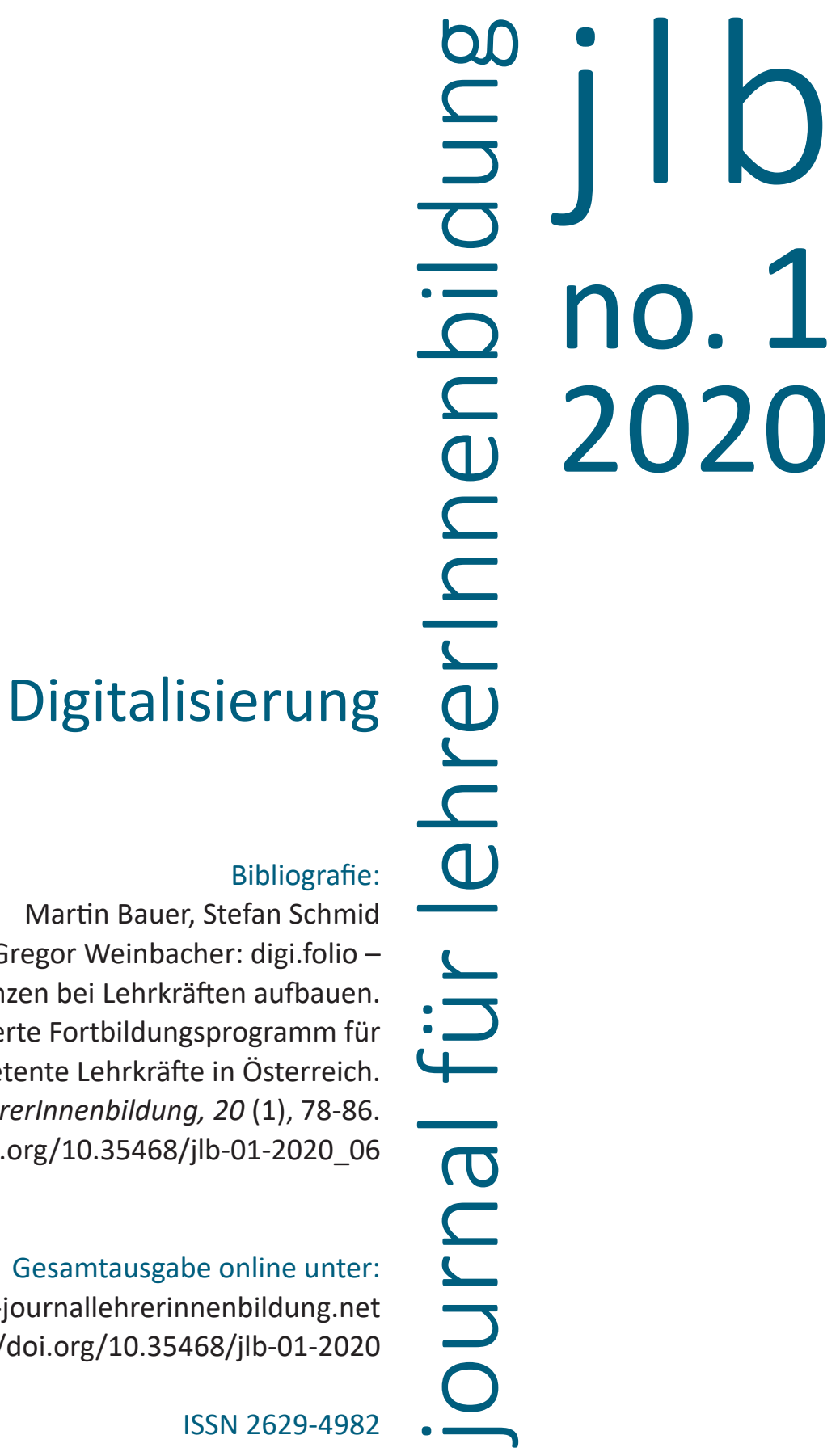


06

Martin Bauer, Stefan Schmid und Gregor Weinbacher

\section{digi.folio - digitale Kompetenzen bei Lehrkräften aufbauen. Das maßgeschneiderte Fortbildungsprogramm für digital kompetente Lehrkräfte in Österreich}




\section{Digitalisierung und Bildung}

Die Digitalisierung macht auch vor dem System Schule nicht halt. Phänomene, Methoden oder Konzepte wie Flipped Learning ${ }^{1}$, Augmented Reality ${ }^{2}$ oder etwa Seamless Learning ${ }^{3}$ gewinnen vor dem Hintergrund der zunehmenden Digitalisierung aller Lebensbereiche auch für schulische Lernprozesse an Bedeutung, werden neu oder völlig neuartig definiert. $^{4}$

Die kontinuierliche und deutliche Zunahme an Computern in Volkschulen innerhalb von 10 Jahren oder etwa der Umstand, dass 2016 in 99,6\% der Bundesschulen in Österreich das Internet im Unterricht genützt wird, belegen einen intensiven Einzug der Informations- und Kommunikationstechnik (IKT) in den Schulalltag (Brandhofer, Baumgartner, Ebner, Köberer, Trültzsch-Wijnen \& Wiesner, 2018).

Dennoch fühlt sich mehr als die Hälfte der Lehrkräfte Österreichs (52\%) gar nicht darauf vorbereitet, IKT im Unterricht zu verwenden. Im EU-Schnitt teilen nur 27\% diese Einschätzung (Schmich, Lindemann \&

1 Als Flipped Classroom wird eine Unterrichtsmethode bezeichnet, bei der vor allem durch den Einsatz digitaler Medien der Unterricht im wahrsten Sinne des Wortes auf den Kopf gestellt wird. Beim klassischen bzw. traditionellen Unterricht findet die Erarbeitung eines Themas oder Stoffes in der Regel im Unterricht statt. Im Unterricht wird Wissen vermittelt - der Großteil der Stunde wird für den Input verwendet. Die Übungsphase kommt oft viel zu kurz und wird - auch aus dem resultierenden Zeitmangel - in die Hausübung verlagert. Beim Konzept Flipped Classroom werden insbesondere Lernvideos anhand deren man ein neues Thema erarbeiten kann, den Lernenden mitgegeben. Diese sehen sich diese Videos zuhause an und erlernen so den neuen Inhalt. Der Input passiert im eigenen Tempo, wann man will und wo man will. Im Unterricht bleibt somit Zeit um Übungen durchzuführen. Die Lehrkraft wird zum Coach und kann individuell unterstützen. Input- und Übungsphase werden also einfach räumlich und zeitlich getauscht, bzw. auf Englisch „flipped“. Ziel des erweiterten Konzeptes Flipped Learning ist den individuellen Lernfortschritt ins Zentrum des Lernprozesses zu stellen.

2 Unter erweiterter Realität, augmented reality oder AR versteht man die computergestützte Erweiterung der Realitätswahrnehmung. Sie kann als Form der Mixed Reality verstanden werden.

3 Im Zentrum des Seamless Learning steht die Verbindung bzw. der durchlässige Übergang von formalen und informellen Lernen. Erst durch die Verbreitung von mobilen Endgeräten rückte das Konzept mit dem Namen „Mobile Seamless Learning" wieder in den Vordergrund. Es beschreibt die Verbindung von mobilen, ortsundabhängigen und durchgängigem Lernen.

4 Zusammenfassend vor allem mit dem Blick auf das österreichische Schulsystem und den digital-innovativen Initiativen, Projekten, Ideen und Konzepten empfiehlt sich die Lektüre von Erziehung \& Unterricht (2017). 
Gurtner-Reinthaler, 2018). Auch in den aktuellen Ausbildungscurricula der Lehramtsstudien in Österreich zeigt sich durchwegs, dass der Bereich "Digital-innovatives Lehren und Lernen" (noch) nicht im gewünschten Maße zu finden ist (Brandhofer et al., 2018), wobei hier angemerkt werden muss, dass seit dieser Feststellung im Nationalen Bildungsbericht zumindest in einem der vier Ausbildungsverbünde Österreichs ${ }^{5}$ informatische und medienpädagogische Grundlagen im Ausmaß von min. $6 \mathrm{EC}$ (Bachelor + Master) verpflichtend verankert wurden (s. Ebner, Adams, Bollin, Kopp \& Teufel, in diesem Heft).

Daher hat das Bundesministerium für Bildung, Wissenschaft und Forschung (BMBWF) im Rahmen des Masterplans für Digitalisierung in der Bildung das Handlungsfeld "Lehrende - Aus-, Fort- und Weiterbildung" neben "Software - Pädagogik, Lehr- und Lerninhalte" sowie „Hardware-Infrastruktur, modernes IT-Management, moderne Schulverwaltung" als zentrales Aktionsfeld verankert (BMBWF, 2019a).

Die darin enthaltene Maßnahme bzw. das Instrument digi.folio bietet allen Lehrpersonen - angehenden sowie im Dienst stehenden - die Möglichkeit für ihren Lehrberuf nötige digitale Kompetenzen maßgeschneidert auf- und auszubauen. digi.folio wurde unter Einbindung aller 14 Pädagogischen Hochschulen Österreichs, insbesondere der Mitglieder der PHELS-Gruppe (E-Learning Strategiegruppe der Pädagogischen Hochschulen Österreichs; BMBWF, 2019b) federführend vom National Center of Competence ( $\mathrm{NCoC}$ ) Virtuelle PH konzipiert und entwickelt. Der inhaltliche Rahmen hierfür bietet das Kompetenzmodell digi.kompP - digitale Kompetenzen für Lehrkräfte.

\section{Digital kompetente Lehrkräfte}

Welche Medienkompetenzen Lehrkräfte unter den Bedingungen und Kontexten der Digitalität vor und während des Studiums sowie in den ersten fünf Berufsjahren erwerben sollten, beschreibt das 2016 im Auftrag des Bundesministeriums für Bildung von der Virtuellen Päda-

5 Aufgrund der Anregungen der 2012 bestehenden Bundesministerien für Bildung und Frauen sowie für Wissenschaft, Forschung und Wirtschaft wurden zur Umsetzung einer gemeinsamen Lehramtsausbildung insgesamt vier Entwicklungsverbünde in Österreich, bestehend aus Universitäten und Pädagogischen Hochschulen, etabliert. 
gogischen Hochschule entwickelte Kompetenzmodell „digi.kompP digitale Kompetenzen für Lehrkräfte“. (vgl. Brandhofer et al., 2016)

Ziel bei der Entwicklung des Modells war es, ein Rahmen- und Referenzmodell zu schaffen, das kompatibel mit international gebräuchlichen Modellen ist und in Inhalt und Logik an bestehende österreichische digi.komp-Modelle für Schülerinnen und Schüler anschließt. digi. kompP wurde basierend auf den Kompetenzmodellen Technological Pedagogical Content Knowledge (Koehler \& Mishra, 2006), ICT Competency Framework for Teachers (United Nations Educational Scientific and Cultural Organization, 2011) und Digital Bildung (Krumsvik, 2014) entwickelt. Das Kompetenzmodell European Framework for the Digital Competence of Educators: DigCompEdu der Europäischen Kommission wurde erst 2017 publiziert und daher bei der aktuell vorliegenden Version 1.0 noch nicht berücksichtigt (Redecker \& Punie, 2017).

digi.kompP beschreibt Can-Do-Statements eingeordnet in acht Kompetenzbereichen, die in den Phasen 0 Vor dem Studium, 1 während des Studiums und 2 während der ersten fünf Berufsjahre, von (angehenden) Lehrkräften erworben werden sollten. digi.kompP stellt demnach das Zielbild einer medienkompetenten Lehrperson im Zeitalter der Digitalität dar.

Das Kompetenzmodell digi.kompP ist zudem auch der inhaltliche Rahmen für jegliche Entwicklungs- und Steuerungsmaßnahmen in der Personalentwicklung von Lehrkräften im Bereich „Digitale Kompetenzen für Lehrkräfte“.

\section{digi.folio - Personalisierter Kompetenzaufbau}

digi.folio ist eine vierstufige Maßnahme und ermöglicht es Lehrkräften maßgeschneidert Medienkompetenzen im Zeitalter der Digitalität aufzubauen. Für Schulleitungen stellt dieses Instrument zudem eine Personalentwicklungsmaßnahme zum gezielten und strategischen Auf- und Ausbau digitaler Kompetenzen des Lehrkörpers dar.

Die Maßnahme digi.folio umfasst

- einen digitalen Kompetenzcheck (digi.checkP),

- eine individuell-maßgeschneiderte Fortbildungsmaßnahme im Ausmaß von $6 \mathrm{EC}$, 
- die Reflexion der eigenen digital-innovativen Lehrtätigkeit in einem Praxisportfolio sowie

- den Nachweis der erworbenen Kompetenzen.

\section{Digitaler Kompetenzcheck (digi.checkP)}

Der digi.checkP ist ein Online-Diagnoseinstrument und gibt Lehrpersonen ein direktes Feedback über deren digitalen Kompetenzstand. digi.checkP besteht aus Reflexions- (Selbsteinschätzungs-) und Wissensfragen zu den acht Bereichen des digi.kompP-Kompetenzmodells. Dieses Feedback unterstützt unter anderem auch die Lehrperson bei der persönlichen Einschätzung, in welchen Bereichen sie schon adäquate digitale Kompetenzen aufweist und in welchen noch ein Kompetenzaufbau - z. B. in Form von Fortbildungslehrveranstaltungen abgestimmt mit der Schulleitung anzustreben sei.

\section{Fortbildungsmaßnahme im Ausmaß von min. 6 EC}

Die im Rahmen der zu besuchenden Lehrveranstaltungen im Ausmaß von min. 6 EC (Workload 150 Stunden) sollen aufgrund der individuellen Ergebnisse im Kompetenzcheck digi.checkP passend gewählt werden können.

„Für die Lehrkräftefort- und -weiterbildung in Österreich sind vor allem die Pädagogischen Hochschulen zuständig. Die Anmeldung zu den angebotenen Lehrveranstaltungen erfolgt über die sog. PH-Online-Portale der Pädagogischen Hochschulen. Das Fort- und Weiterbildungsangebot wird demzufolge in 14 verschiedenen $\mathrm{PH}-$ Online-Systemen verwaltet und auf unterschiedliche Weisen - zum Beispiel auf einzelnen Webseiten, in Newslettern oder spezifischen Dokumenten - dargestellt." (Schmid, 2017, S. 16)

Im Rahmen der Maßnahme digi.folio wird das Angebot der relevanten Lehrveranstaltungen aller österreichischen Pädagogischen Hochschulen auf der Webseite https://www.digifolio.at/veranstaltungen/ automatisiert zusammengeführt. Userinnen und User können hier im Anschluss an den digi.checkP nach für sie relevanten Lehrveranstaltungen mit Filtermöglichkeit nach Pädagogische Hochschule und digi. kompP-Bereich suchen und sich für gewünschte Lehrveranstaltungen im jeweiligen PH-Online-Portal anmelden. Zudem besteht auch die Möglichkeit nach Gestaltungsart der Fortbildung zu filtern: Blended Learning, Präsenzlehre oder Online. „Vor allem vor dem Hintergrund der zunehmenden Individualisierung und Personalisierung im Sinne 
einer Anpassung der Bildungsangebote an die eigenen Bedürfnisse und Erfordernisse zeigt sich die Relevanz ..." (ebd., S. 17) dieser im responsiven Design zusammengefassten Darstellung aller relevanten Lehrveranstaltungen aller Pädagogischer Hochschulen.

\section{Reflexion der eigenen digital-innovativen Lehrtätigkeit}

Im Rahmen eines persönlich geführten ePortfolios wird die durch die Fortbildungen geänderte Lehr- und Lerntätigkeit reflektiert. Damit wird der Praxistransfer ins Zentrum der Maßnahme gerückt.

\section{Nachweis der erworbenen Kompetenzen}

Zum Abschluss der Maßnahme können die Teilnehmenden in der Plattform digi.folio nach erfolgtem Login ihr digi.checkP-Ergebnis, ihre Teilnahmebestätigungen der absolvierten Lehrveranstaltungen (Fortbildungen an Pädagogischen Hochschulen) im Mindestausmaß von 6 EC sowie einen Link zu einem persönlich geführten Portfolio, wo die eigene digital-innovative Lehrpraxis dokumentiert wird, hochladen.

In einem anschließenden Reflexionsgespräch mit der Schulleitung wird die Maßnahme abgeschlossen. Vor allem durch diesen letzten Schritt wird die Maßnahme zum Personalentwicklungsinstrument.

\section{Das aktuelle Fortbildungsangebot}

Seit März 2019 wurden an den Pädagogischen Hochschulen insgesamt 690 Fortbildungsmaßnahmen in diesem Bereich angeboten. Von diesen Veranstaltungen wurde mehr als die Hälfte (53\%) in einem Blended Learning-Format angeboten. 19\% der Fortbildungen finden rein online, $28 \%$ in einem reinen Präsenzformat statt.

Unter zu festigenden Kompetenzen wurden vor allem Lehrveranstaltungen angeboten, die in die Kompetenzbereiche $C$ (Digital Materialien Gestalten), D (Digital Lehren und Lernen) und $\mathrm{E}$ (Digital Lehren und Lernen im Fach) fallen. Auf jeden dieser drei Kompetenzbereiche entfallen jeweils mehr als 40\% der seit März 2019 absolvierten Maßnahmen. Mit knapp 52\% am häufigsten angeboten wurden Fortbildungen aus dem Kompetenzbereich $\mathrm{E}$. Hierbei ist zu beachten, dass einer Veranstaltung mehrere Kompetenzbereiche gleichzeitig zugeordnet sein können (so kann eine Fortbildung etwa die Kompetenzbereiche B, C 
und $\mathrm{E}$ abdecken), weswegen diese Prozentwerte nicht einfach aufsummiert werden können.

\section{Conclusio und Ausblick}

Die vorliegenden Veranstaltungszahlen legen nahe, dass die Pädagogischen Hochschulen bereits viele Fortbildungen zum Aus- und Aufbau digitaler Kompetenzen für Lehrkräfte anbieten.

Durch das vorangehende Online-Diagnoseinstrument digi.checkP sollen Lehrpersonen dabei unterstützt werden für sie passende Lehrveranstaltungen zu finden. Zudem bietet eine systematische Auswertung der digi.checkP-Ergebnisse den Pädagogischen Hochschulen die Möglichkeit Fortbildungen mit den notwendigen Inhalten anzubieten. Im Rahmen einer oberösterreichweiten Pilottestung im November 2017 mit über 21.000 teilnehmenden Lehrkräften wurde der digi.checkP erstmals großflächig eingesetzt. Die Ergebnisse gaben Aufschluss über einen möglichen Fortbildungsbedarf im Bundesland Oberösterreich und flossen bzw. fließen in die Angebots- und Seminargestaltung der $\mathrm{PH}$ Oberösterreich mit ein.

Sehr spannend scheint auch, dass weniger als ein Drittel der angebotenen Fortbildungsveranstaltungen in reiner Präsenzlehre angeboten werden. Mehr als die Hälfte aller Veranstaltungen wird als Blended Learning und bereits $19 \%$ als reine Online-Bildungsangebote durchgeführt. Bei letzterem finden sich vor allem kooperative Online-Seminare.

Die erst kürzlich durchgeführte curriculare Verankerung von informatischen und medienpädagogischen Inhalten bzw. zu erwerbenden Kompetenzen in den Lehramts-Bachelor- und Master-Studien von insgesamt mindestens 6 EC im Ausbildungsverbund Süd-Ost zeigt eine Anpassung bzw. Veränderung im Ausbildungsbereich und verschiebt zukünftig zumindest einen Teil des (theoretischen) Kompetenzerwerbs von der Fort- und Weiterbildung in die Erstausbildung. Der gewünschte, für die Maßnahme mehr als relevante und im ePortfolio dokumentierte Praxistransfer macht digi.folio dennoch zu einem Instrument der Fortbildung sowie standortspezifischen Personal- und Professionsentwicklung.

Lediglich die Anpassung der Curricula der Lehramtsstudien ist demzufolge nicht ausreichend, da garantiert sein muss, dass auch bereits im 
Dienst stehende Lehrkräfte adäquat auf die Herausforderungen der Digitalisierung vorbereitet werden und der Praxistransfer zu einer der Zeit passenden Transformation des Bildungs-, Schul- und Unterrichtsgeschehen führt.

digi.folio wurde bereits per Rundschreiben des Bundesministerium für Bildung, Wissenschaft und Forschung im Jahr 2019 allen Personen der Schulaufsicht - insbesondere auch den Schulleitungen - als Instrument des standortspezifischen digitalen Kompetenzauf- und -ausbaus des Lehrkörpers zur Kenntnis gebracht. Weitere bewusstseinsbildende und unterstützende Maßnahmen sowie zirkuläre Feedbackschleifen sind anzustreben.

\section{Literatur}

BMBWF. (2019a). Digitale Bildung. Masterplan für die Digitalisierung im Bildungswesen. Verfügbar unter https://www.bmbwf.gv.at/Themen/schule/zrp/dibi.html [18.11.2019].

BMBWF. (2019b). Die PHELS. Die E-Learning Strategiegruppe der Pädagogischen Hochschulen Österreichs. Verfügbar unter https://www.bmbwf.gv.at/Themen/ schule/zrp/dibi/paed/phels.html [18.11.2019].

Brandhofer, G., Baumgartner, P., Ebner, M., Köberer, N., Trültzsch-Wijnen, C. \& Wiesner, C. (2018). Bildung im Zeitalter der Digitalisierung. In S. Breit, F. Eder, K. Krainer, C. Schreiner, A. Seel \& C. Spiel (Hrsg.), Nationaler Bildungsbericht Österreich 2018 (Bd. 2) (S. 307-362). Verfügbar unter https://www.bifie.at/wp-content/uploads/ 2019/03/NBB_2018_Band2_Beitrag_8.pdf [20.01.2020].

Brandhofer, G., Kohl, A., Miglbauer, M. \& Narosy, T. (2016). digi.kompP - Digitale Kompetenzen für Lehrende. Das digi.kompP-Modell im internationalen Vergleich und in der Praxis der österreichischen Pädagoginnen- und Pädagogenbildung. R\&E Source, 6, 38-51. Verfügbar unter https://journal.ph-noe.ac.at/index.php/resource/ article/view/305/381 [22.01.2020].

Erziehung \& Unterricht (2017). Österreichische Pädagogische Zeitschrift. Lernen und Lehren mit Technologien: Vermittlung digitaler und informatischer Kompetenzen, 167 (7-8).

Koehler, M. \& Mishra, P. (2006). Technological Pedagogical Content Knowledge: A Framework for Teacher Knowledge. Teachers College Record, 108 (8), 1017-1054.

Krumsvik, R. J. (2014). Teacher educators' digital competence. Scandinavian Journal of Educational Research, 58 (3), 269-280.

Redecker, C. \& Punie, Y. (2017). European Framework for the Digital Competence of Educators DigCompEdu. Verfügbar unter https://ec.europa.eu/jrc/en/publication/ eur-scientific-and-technical-research-reports/european-framework-digital-competence-educators-digcompedu [18.11.2019].

Schmich, J., Lindemann, R. \& Gurtner-Reinthaler, S. (2018). Aus- und Fortbildung von Lehrkräften und Schulleitungen. In J. Schmich \& U. Itzlinger-Bruneforth (Hrsg.), TALIS 2018 - Rahmenbedingungen des schulischen Lehrens und Lernens aus Sicht 
von Lehrkräften und Schulleitungen im internationalen Vergleich (Bd. 1) (pp. 3952). Graz: Leykam.

Schmid, S. (2017). digifolio und Bildungsmarketing!? Forum neue Medien in der Lehre Austria $<$ fnm > magazin, 4, 15-17. Verfügbar unter https://www.fnma.at/content/ download/713/2511 [20.01.2020].

United Nations Educational Scientific and Cultural Organization. (2011). UNESCO ICT Competency Framework for Teachers. Verfügbar unter https://unesdoc.unesco. org/ark:/48223/pf0000213475 [21.01.2020].

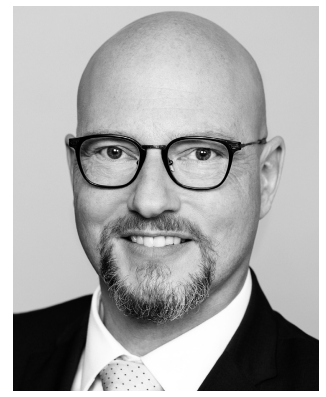

Martin Bauer, Mag., MSc,

Leiter der Abteilung Präs/15 - IT-Didaktik

im Bundesministerium für Bildung, Wissenschaft und Forschung Österreich.

Arbeitsschwerpunkt:

Digitalisierung im Bildungswesen

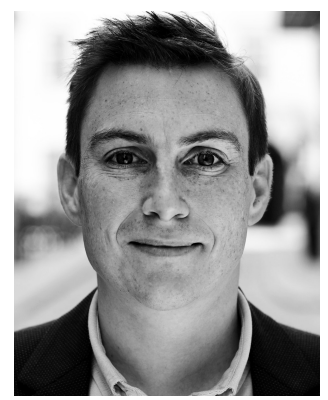

Stefan Schmid, Mag., BEd, Sachbearbeiter in der Abteilung Präs/15 - IT-Didaktik im Bundesministerium für Bildung, Wissenschaft und Forschung Österreich.

Arbeitsschwerpunkt:

Digital-innovative Lehrer*innenbildung

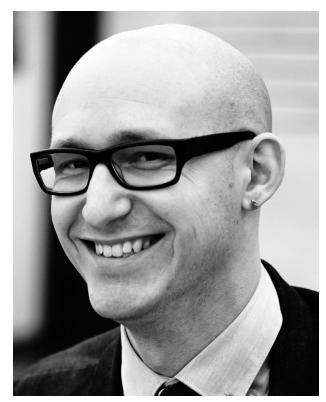

Gregor Weinbacher, MA, BA, BA,

Mitarbeiter in der Abteilung Präs/15 - IT-Didaktik im Bundesministeriums für Bildung, Wissenschaft und Forschung Österreich.

Arbeitsschwerpunkte:

Begutachtung und Einschätzung von Bildungstechnologien sowie Planung und Steuerung im Zusammenhang mit Softwarelizenzen für österreichische Bundesschulen

Gregor.Weinbacher@bmbwf.gv.at 